\title{
Establishment of an Experimental Breast Cancer ZHENG Model and Curative Effect Evaluation of Zuo-Jin Wan
}

\author{
Jia Du, Yang Sun, Xiu-Feng Wang, Yi-Yu Lu, Qian-Mei Zhou, and Shi-Bing Su \\ Research Center for Traditional Chinese Medicine Complexity System, Shanghai University of Traditional Chinese \\ Medicine, 1200 Cailun Road, Pudong, Shanghai 201203, China
}

Correspondence should be addressed to Shi-Bing Su; shibingsu07@163.com

Received 18 August 2013; Accepted 22 October 2013

Academic Editor: Aiping Lu

Copyright ( 2013 Jia Du et al. This is an open access article distributed under the Creative Commons Attribution License, which permits unrestricted use, distribution, and reproduction in any medium, provided the original work is properly cited.

\begin{abstract}
Herbal formulas based on the traditional Chinese medicine (TCM) syndrome (ZHENG) have been used as alternative treatments for breast cancer. However, there is a lack of the experimental animal ZHENG model for the evaluation of the herbal formulas. In this study, we have established 4T1 mouse breast cancer with Liver Fire Invading Stomach Syndrome model (4T1 LFISS mice) and investigated the effects of the herbal formula, Zuo-Jin Wan (ZJW). Our results showed that 4T1 LFISS mice have the features of LFISS including irritability, loss of appetite, yellow urine, chow, and a tail hot. Compared to untreated 4T1 LFISS mice, ZJW significantly reduced tumor weight and volume $(P<0.05)$, although it was weaker than Cisplatin. However, ZJW significantly increased the body weight and food intake of 4T1 LFISS mice and decreased serum ALT, AST, Cr, and BUN levels and ZHENG score $(P<0.05)$, while Cisplatin reduced the food intake, and body weight and increased serum ALT, AST, Cr, and BUN levels in 4T1 LFISS mice. Our study has provided a mouse breast cancer ZHENG model and showed that ZJW suppresses tumor growth and improves LFISS and kidney and liver functions in the 4T1 LFISS mice.
\end{abstract}

\section{Introduction}

Breast cancer is the most common cancer among women; 1.38 million women were diagnosed with breast cancer in 2008. Its incidence rates vary considerably, with the highest rates in Europe and the lowest rates in Africa and Asia [1]. Its incidence rate in China is also increasing rapidly, and the number of cases increased by $38.5 \%$ from 2000 to 2005 [2]. However, there currently is a lack of clinically safe and effective drugs for prevention and treatment of breast cancer. In traditional Chinese medicine (TCM), some herbal formulas originating from natural products have been used as alternative treatments for breast cancer [3].

In order to experimentally evaluate the effectiveness and safety of treatment based-ZHENG (TCM syndrome) for cancer, an animal ZHENG model is necessary. However, there is a lack of an experimental animal ZHENG model in cancer research. Recently, Chen et al. [4] have established mouse xenograft pancreatic cancer models with dampnessheat, spleen-deficiency, and blood-stasis syndromes and found that they correlated with the treatment response of herbal medicine. An animal ZHENG models for breast cancer needs to be established for the evaluation of Chinese herbal formula.

Zuo-Jin Wan (ZJW, also called Zuo-Jin pill) is a Chinese herbal formula. In the clinical practices of TCM, the efficacy of ZJW is based on Liver Fire Invading Stomach Syndrome (LFISS, a ZHENG), which is characterized by a choking sensation in the chest and irritability, loss of appetite, yellow urine, red tongue, and more in patients with cancer. Moreover, in recent studies, ZJW has been identified experimentally to have anticancer activity in recent studies in gastric cancer [5], liver cancer [6] and colorectal cancer [7], 
TABLE 1: Semiquantitative evaluation of symptoms and signs.

\begin{tabular}{|c|c|c|c|}
\hline Symptoms & Light (1 point) & Middle (2 points) & Heavy (3 points) \\
\hline Irritability & $\begin{array}{l}\text { Angry, irritable, and occasional } \\
\text { mood swings }\end{array}$ & $\begin{array}{l}\text { Angry, easily irritable, but can be } \\
\text { controlled }\end{array}$ & $\begin{array}{l}\text { Often be agitated and angry, and } \\
\text { difficult to control }\end{array}$ \\
\hline Loss of appetite & $\begin{array}{l}\text { Poor appetite, amount eaten } \\
\text { decreases by less than a third of } \\
\text { original amount }\end{array}$ & $\begin{array}{l}\text { Appetite reduction, amount eaten } \\
\text { decreases by more than a third }\end{array}$ & No appetite, eat less than half \\
\hline Yellow urine & Slightly yellow urine & Yellow urine & Deep yellow urine \\
\hline Claws and tail hot & Claws and tail slightly hot & Claws and tail hot & Claws and tail very hot \\
\hline
\end{tabular}

and multiple cancer cell lines, and it has suggested that the anti-cancer activities are due to induction of mitochondriadependent apoptosis pathway [8]. However, whether ZJW has inhibitory activities against tumor growth without the side effects in breast cancer is unknown.

In this study, we have established $4 \mathrm{~T} 1$ mouse breast cancer with Liver Fire Invading Stomach Syndrome model (4T1 LFISS mice) and investigated the effects and safety of ZuoJin Wan (ZJW) compared to Cisplatin on 4T1 LFISS mice. Our results showed that ZJW inhibits the tumor growth and improves LFISS and kidney and liver functions in the 4T1 LFISS mice.

\section{Materials and Methods}

2.1. Cell Culture. The mouse $4 \mathrm{~T} 1$ mammary tumor cell line was purchased from Shanghai Cell Bank, Chinese Academy of Sciences (Shanghai, China), and was cultured in DMEM medium (Gibco, San Francisco, CA, USA) supplemented with $10 \%$ heat-inactivated $\left(56^{\circ} \mathrm{C}, 30 \mathrm{~min}\right)$ fetal calf serum (PAA, A-4061, Pasching, Austria), penicillin (100 U/mL), and streptomycin $(100 \mu \mathrm{g} / \mathrm{mL})$. The cell culture was maintained at $37^{\circ} \mathrm{C}$ with $5 \% \mathrm{CO}_{2}$ in a humidified atmosphere.

2.2. 4 T1 LFISS Mice. Female BALB/c mice (5 weeks old) were purchased from SLAC Laboratory Animal (Shanghai, China) and bred in the Laboratory Animal Center at Shanghai University of traditional Chinese medicine. The mice were housed in pathogen-free condition throughout the experimental duration and given free access to commercial rodent chow and water. $4 \mathrm{~T} 1$ cells $\left(3 \times 10^{6}\right.$, suspended in $100 \mu \mathrm{L}$ of PBS), were injected into mammary fat pads of female BALB/c mice.

2.3. Preparation of Zuo-Jin Wan and Cisplatin. Zuo-Jin Wan is formulated by mixing the herbs, Rhizoma Coptidis and Fructus Euodiae as 6:1 ratio. The herbs were purchased from Shanghai Kangqiao Chinese Traditional Medicine Co., Ltd. (Shanghai, China). The aqueous extracts of these herbs was prepared and the quality control was performed as described previously [4]. The extract was stored at $-20^{\circ} \mathrm{C}$, and its preparations were standardized, regulated, and quality controlled according to the guidelines defined by the Chinese State Food and Drug Administration. Cisplatin injection was purchased from Nanjing Pharmaceutical Factory Co., Ltd. (Nanjing, China).
2.4. Treatment Protocol. One day after tumor cell inoculation, the mice were randomly divided into four groups $(n=8$ per group; normal, $n=6)$. ZJW-treated group $(1.8 \mathrm{~g} / \mathrm{kg}$, once a day) was received by gavages, and Cisplatin-treated group ( $5 \mathrm{mg} / \mathrm{kg}$, next day at a time), the positive control, was received by intraperitoneal injection as a positive control. Untreated groups were divided into a normal group and a model group. The model group was received with physiological saline as a sham control. The treatments lasted for 3 weeks. The mouse body weight, food intake, tumor weight, and volume were measured at different time points following tumor implantation. The tumor volume $(V)$ was calculated by $V=a$ (long diameter $) \times b$ (short diameter) $2 \div 2$.

2.5. Liver and Kidney Functions Tests. After obtaining blood samples by picked eyeballs of the mice, it was centrifuged at $3000 \mathrm{rpm}$ for 10 minutes in order to separate and collect the serum. Serum alanine transaminase (ALT), aspartate transaminase (AST), serum creatinine (Cr), and blood urea nitrogen (BUN) were measured using the colorimeter testing kit (Kangcheng, Nanjing, China). According to the manufacturer's instructions, serum samples were measured at $510 \mathrm{~nm}$, $510 \mathrm{~nm}, 510 \mathrm{~nm}$, and $520 \mathrm{~nm}$, respectively.

2.6. Evaluation of ZHENG in Mice. The LFISS, a ZHENG, in $4 \mathrm{~T} 1$ mice was diagnosed by its characteristic symptoms and signs, which include irritability, loss of appetite, yellow urine and claws, and a tail hot. It was given a semiquantitative evaluation as shown in Table 1, according to the established methodology and criteria for ZHENG animal models [9].

2.7. Efficacy Evaluation of ZHENG. The efficacy evaluation of ZHENG in mice was done according to the "Guideline for Clinical New Drug Research in Chinese Herbal Medicine" [10]. The standard of ZHENG outcome was scored as follows: ZHENG score as none: 0; light: 1 point; middle: 2 points; heavy: 3 points (Table 1). The calculation formula was as follows: the efficacy index of ZHENG $(N)=[$ (before treatment score - after treatment score)/before treatment score] $\times$ $100 \%$. The efficacy evaluation standard of ZHENG was the following: experimental cure: $N \geq 90 \%$; excellent: $N<90 \%$ to $60 \%$; effective: $N \leq 60 \%$ to $>30 \%$; invalid: $N \leq 30 \%$ [11].

2.8. Statistical Analyses. All data was expressed as means \pm SD. Comparisons between groups were performed by 


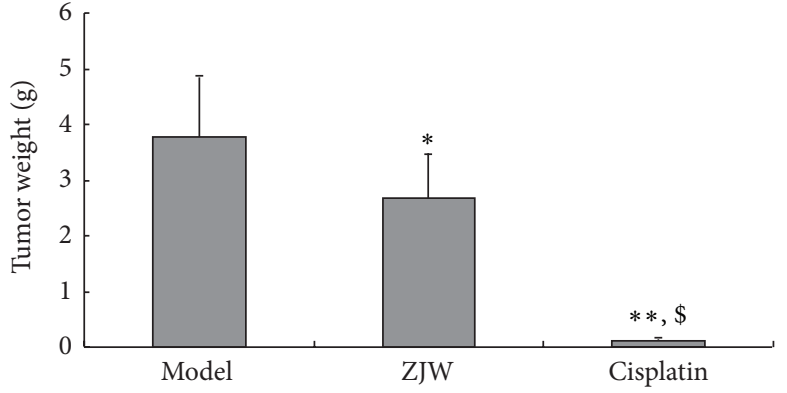

(a)

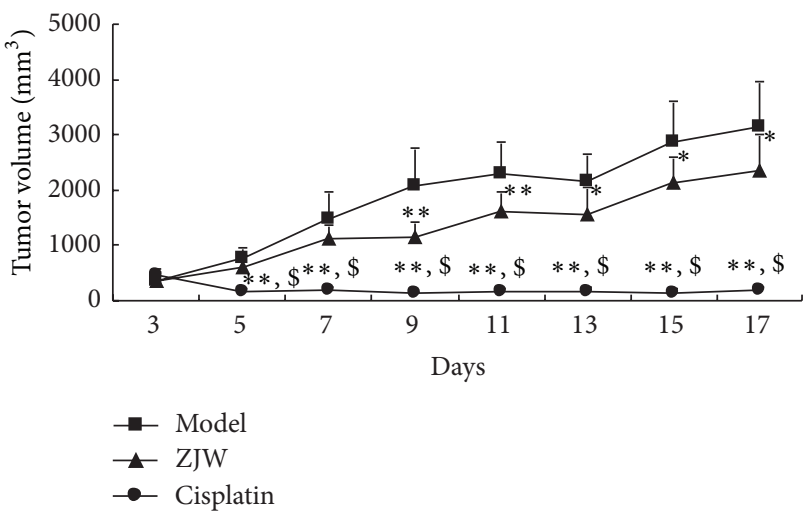

(b)

FIGURE 1: ZJW reduced tumor weight and volume in 4T1 LFISS mice. Tumor weights (a) and volumes (b) in 4T1 LFISS mice with ZJW and Cisplatin in treatments were measured, respectively. ${ }^{*} P<0.05,{ }^{* *} P<0.01$, versus model group; ${ }^{\circledR} P<0.05$, versus ZJW-treated group.

Student's $t$-test and one-way analysis of variance (ANOVA). The level of significance was set at $P<0.05$.

\section{Results}

3.1. ZJW Reduced Tumor Weight and Volume in 4T1 LFISS Mice. To determine whether ZJW could suppress the tumor growth of breast cancer, we tested the ability of ZJW to reduce tumor weight and volume in 4T1 LFISS mice. The tumor weights were significantly reduced in the ZJW-treated group $(P<0.05)$ and the Cisplatin-treated group $(P<0.01)$ compared to the model group, respectively (Figure 1(a)). Moreover, the tumor volumes were significantly reduced in the ZJW-treated group on days 9 and $11(P<0.01)$ and on days 13,15 , and $17(P<0.05)$. It significantly reduced on 5 to 17 days $(P<0.01)$ in the Cisplatin-treated group compared to the model group (Figure 1(b)). There were significant differences between the ZJW-treated group and the Cisplatintreated group $(P<0.05)$. Together, these results suggested that ZJW can suppress the tumor growth, although it was weaker than Cisplatin in 4T1 LFISS mice.

3.2. Effects of ZJW on Body Weight in 4T1 LFISS Mice. In order to detect whether ZJW has any side effects, we measured the body weight of 4T1 LFISS mice once each day. As shown in Figure 2(a), there were no significant differences of the body weight among normal group, model groups and ZJW-treated group $(P>0.05)$. However, starting from 3 rd day, there were significant differences between Cisplatin-treated group $(P<$ 0.01 ) and the other groups.

\subsection{Effects of ZJW on ALT, AST, Cr, and BUN in 4T1 LFISS} Mice. We further tested whether ZJW has any effect on liver and kidney functions. The results showed that ALT (Figure 3(a)), AST (Figure 3(b)), Cr (Figure 3(c)), and BUN (Figure 3(d)) significantly increase in model group compared to normal group $(P<0.05)$. ALT (Figure 3(a)) and $\mathrm{Cr}$

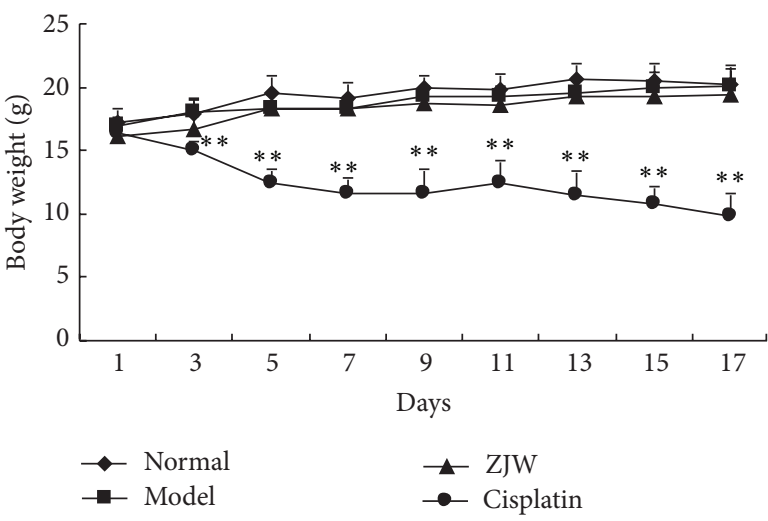

FIGURE 2: Effects of ZJW on body weight in 4T1 LFISS mice. Mice were treated by ZJW and Cisplatin. The body weights of 4T1 LFISS mice at different time points were measured. ${ }^{* *} P<0.01$, versus model group.

(Figure 3(c)) were significantly decreased in ZJW-treated group compared to model group $(P<0.05)$, but there were no significant changes in the level of AST (Figure 3(b)) and BUN (Figure 3(d)) $(P<0.05)$. ALT (Figure 3(a)), AST (Figure 3(b)), Cr (Figure 3(c)), and BUN (Figure 3(d)) significantly increased in Cisplatin-treated group compared to model group $(P<0.05)$. Moreover, there were significant differences between ZJW-treated group and Cisplatin-treated group $(P<0.01)$. Together, these results suggested that ZJW has no side effects and improves liver and kidney functions while Cisplatin reduces body weight and increases ALT, AST, $\mathrm{Cr}$, and BUN in 4T1 LFISS mice.

3.4. ZJW Increased Food Intake in 4T1 LFISS Mice. In order to detect whether ZJW increased food intake, we measured the food intake of 4T1 LFISS mice once every 2 days. As shown in Figure 4, the food intake of 4T1 LFISS mice was significantly decreased in model group compared to normal 


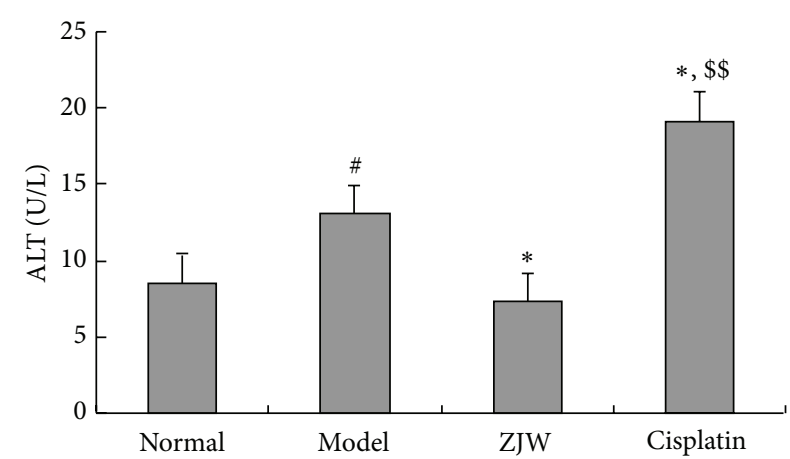

(a)

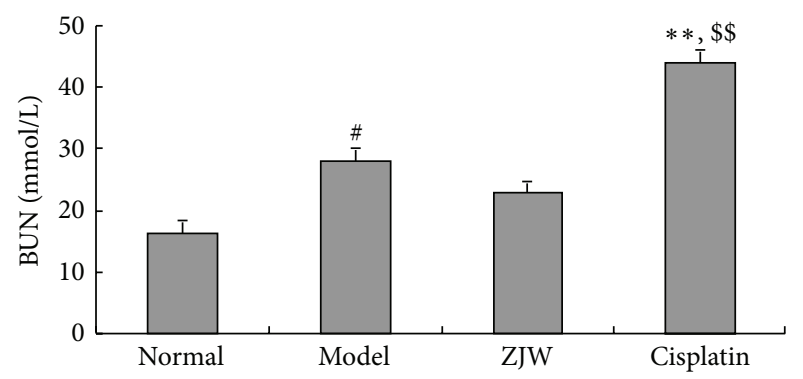

(c)

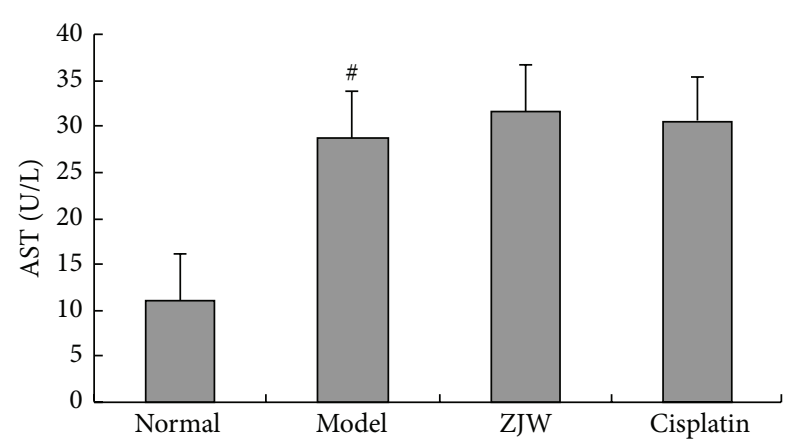

(b)

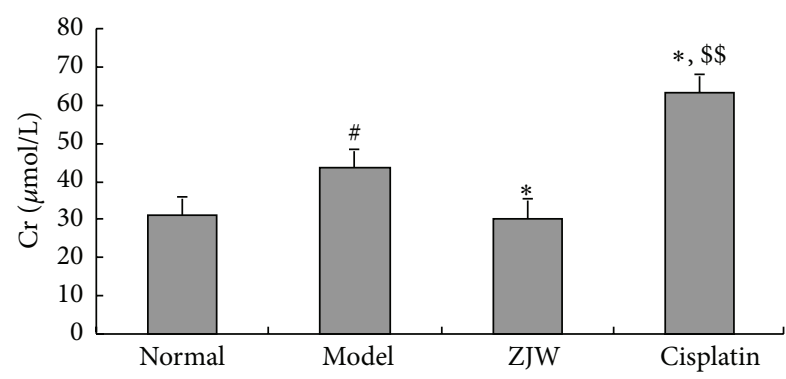

(d)

FIGURE 3: Effects of ZJW on kidney and liver functions in 4T1 LFISS mice. Mice were treated by ZJW and Cisplatin. (a) ALT, (b) AST, (c) BUN, and (d) Cr were measured using the colorimeter testing kit. ${ }^{*} P<0.01$, versus model group; ${ }^{\#} P<0.05$, versus normal group; ${ }^{\$ \$} P<0.01$, versus ZJW-treated group.

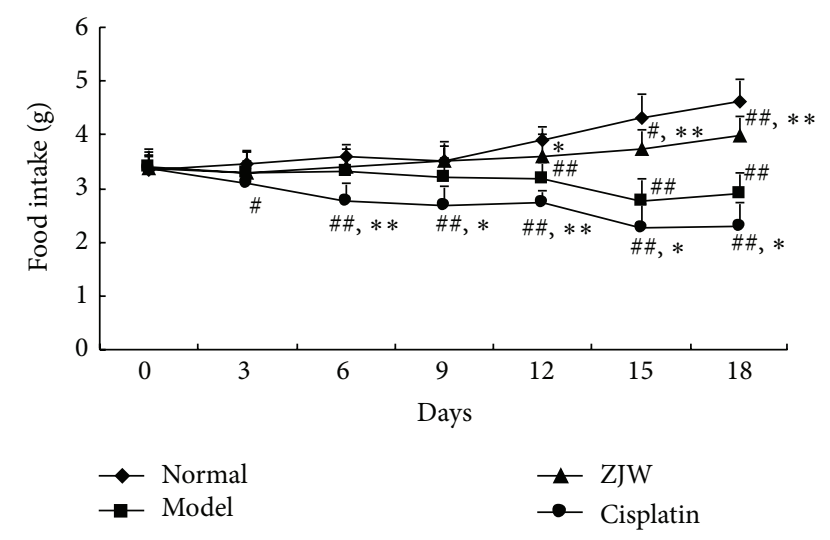

FIgURE 4: Effect of ZJW on food intake in 4T1 LFISS mice. The food intake in 4T1 LFISS mice with ZJW and Cisplatin treatments was measured. ${ }^{*} P<0.05,{ }^{* *} P<0.01$, versus model group; ${ }^{\#} P<$ $0.05,{ }^{\# \#} P<0.01$, versus normal group; ${ }^{\$} P<0.05,{ }^{\$} P<0.01$, versus ZJW-treated group.

group on days 12,15, and 18, respectively $(P<0.05)$. Moreover, the food intake was significantly increased in ZJWtreated group compared to model group $(P<0.05$ or $P<$ 0.01 ) from the 15 th day after treatment, but the food intake in Cisplatin-treated group was not only less than model group but was also significantly different from ZJW-treated group and normal group $(P<0.01)$. The results suggest that ZJW increased food intake while Cisplatin reduces food intake in 4T1 LFISS mice.
3.5. ZJW Decreased ZHENG Score in 4T1 LFISS Mice. To detect whether ZJW impacted the quality of life of the animals compared to Cisplatin treatment, we measured the ZHENG score of 4T1 LFISS mice once every 2 days. As shown in Figure 5, the ZHENG scores were significantly decreased in ZJW-treated group compared to model group $(P<0.05$ or $P<0.01)$ from the 12 th day after treatment, but Cisplatin-treated group was not significantly different from model group $(P<0.05)$, and the ZHENG scores were significantly decreased in Cisplatin-treated group compared to ZJW-treated group at day 15 and day 18 , respectively $(P<$ $0.01)$. The efficacy index of ZHENG is $53 \%$ in ZJW-treated group and $6.2 \%$ in Cisplatin-treated group, indicating that the ZJW treatment was effective and Cisplatin treatment was invalid. The results suggest that ZJW improves the quality of life of 4T1 LFISS mice through the decrease of ZHENG score while Cisplatin is unable to reduce ZHENG score in $4 \mathrm{~T} 1$ LFISS mice.

\section{Discussion and Conclusions}

Treatment based on ZHENG differentiation, also called "Bian Zheng Shi Zhi", is the comprehensive analysis of clinical information that is used to guide the choice of treatment with TCM herbal formulae $[11,12]$. It is the main approach in the clinical practice to increase the effectiveness and safety of TCM treatment in the clinical practice. Following the revaluation of herbal formulas-based ZHENG, the development of new drugs and the discovery of the mechanisms 


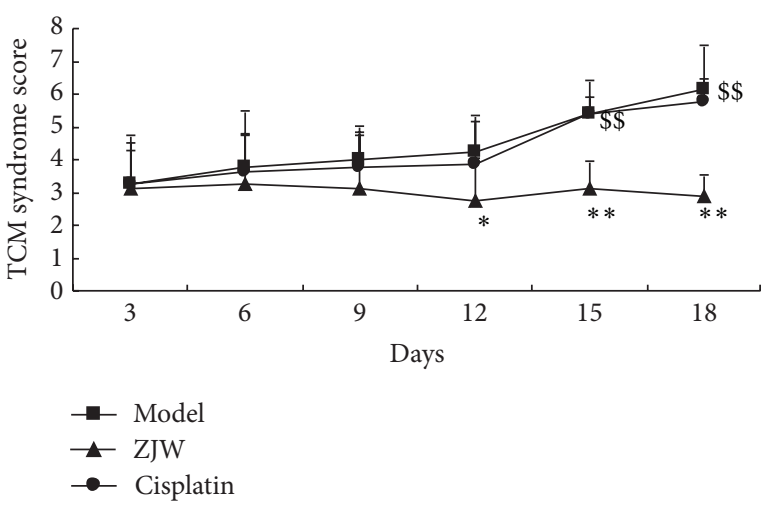

FIGURE 5: ZJW decreased ZHENG score in 4T1 LFISS Mice. The ZHENG scores in 4T1 LFISS mice with ZJW and Cisplatin treatments were measured. ${ }^{*} P<0.05,{ }^{* *} P<0.01$, versus model group; ${ }^{\$ \$} P<0.01$, versus ZJW-treated group.

all need an experimental animal model. Therefore, making the experimental animal ZHENG model as well as an animal disease model is important to breast cancer research.

The animal ZHENG model is necessary for ZHENG research; however, there is a lack of the experimental animal ZHENG model in mice. Recently, Chai et al. have established a Deficiency of both Qi and Yin Syndrome (DQYS) model with the clinical features and one key pathological factor in mice [13]. Previous studies have also established mouse pancreatic cancer models for evaluation of the molecular mechanisms underlying ZHENG and tumor growth [14], and the administration of herbal medicine to the ZHENG model modified the tumor microenvironment [4]. In this study, a mouse breast cancer ZHENG model was established. It was 4T1 mouse mammary cancer ZHENG (LFISS) model, that is, 4T1 LFISS mice. The symptomatic features of 4T1 LFISS mice were irritability and loss of appetite, yellow urine, hot tails and/or claws, along with increase in tumor size (Figure 1), decrease in body weights (Figure 2), and liver and kidney functions disorders (Figure 3). Furthermore, it was found that the ZHENG of 4T1 LFISS mice responds to the ZJW treatment and does not responded to Cisplatin treatment, indicating that the established $4 \mathrm{~T} 1$ mouse breast cancer LFISS model is useful for the evaluation of the efficacy of herbal formulas.

Cisplatin is a common chemotherapy drug for breast cancer therapy, but its side effects such as nephrotoxicity [15], myelotoxicity [16], and neurotoxicity [17] limit its use. In this study, although Cisplatin treatment significantly suppressed tumor growth better than ZJW (Figure 1), it resulted in a decrease in body weight (Figure 2), liver and kidney functions disorders (Figure 3), and reduction in food intake (Figure 4). On the contrary, although the effect on inhibitory tumor is less than that of Cisplatin, ZJW increased body weight and food intake (Figures 2 and 4), improved liver and kidney functions (Figure 3), and improved the quality of life by reducing the ZHENG score (Figure 5) in 4T1 LFISS mice. It suggested that ZJW may be useful the as alternative treatment for breast cancer. Further research will investigate the effects of ZJW combined with Cisplatin.
Recurrently, the use of natural Chinese herbal medicine with antitumor effects is receiving to the treatment of breast cancer [18]. It is a holistic approach through multilevel, multitarget and multi-channel control, which focuses on reducing the side effects of chemotherapy, reversing drug resistance, and improving the quality of life and survival of patients. Therefore, these unique advantages have gradually made the TCM approach more promising in combating breast cancer $[3,19,20]$. Previous studies have shown that some herbal formulas have been used for the treatment of breast cancer including PC-SPESII extract [21] and Sangu decoction [20]. Although ZJW has been first found to have the antitumor effects on breast cancer in this study, the underlying mechanisms remain unknown.

ZJW consists of a combination of two Chinese herbs, Rhizoma Coptidis and Fructus Evodiae, in the ratio of $6: 1$ (w/w) $[4,22,23]$, which contains high levels of active antineoplastic compounds such as berberine and evodiamine [24]. It has been reported that Rhizoma Coptidis and Fructus Evodiae synergistically inhibit s180 tumors in vivo [23], and the synergy of berberine and evodiamine enhances apoptosis of human hepatocellular carcinoma SMMC-7721 cells [24]. Moreover, it has been reported that evodiamine induces some cancer cell apoptosis, such as in human melanoma A375-S2 cells [25], human colorectal carcinoma COLO-205 cells [26], and human breast cancer MDA-MB-231 cells [25]. In addition, previous studies have shown that ZJW can inhibit the expression of inflammatory mediators [27] and modulate the monoaminergic neurotransmitter system [28] and catecholamine secretion [22]. These results indicated that the effects of ZJW may be involved in the induction of cancer cell apoptosis and the adjustment of the nerve, endocrine, and immune systems.

In conclusion, the present study is aiming to establish a 4T1 mouse breast cancer ZHENG model and investigate the antitumor effects of the herbal formula, ZJW. Our study has established a mouse breast cancer ZHENG model and showed that ZJW suppresses tumor growth and improves the quality of life through reducing ZHENG score, improving the kidney and liver functions and without obvious side effects in the 4T1 LFISS mice. The results indicated that ZJW may be useful as alternative treatment for breast cancer. However, further research is needed to investigate the mechanisms of ZJW efficacy and safety.

\section{Conflict of Interests}

All authors declared that there is no conflict of interests.

\section{Authors' Contribution}

Jia Du and Yang Sun equally contributed to this research.

\section{Acknowledgments}

This study was supported by the National Natural Science Foundation (81073134), “085" First-Class Discipline 
Construction Innovation Science and Technology Support Project of Shanghai University of TCM (085ZY1206) and the E-institutes of Shanghai Municipal Education Commission (E 03008).

\section{References}

[1] J. Ferlay, H. R. Shin, F. Bray et al., GLOBOCAN, 2008 V1. 2, Cancer Incidence and Mortality Worldwide: IARC Cancer Base No. 10 [Internet], International Agency for Research on Cancer, Lyon, France, 2010, http://globocan.iarc.fr/.

[2] L. Yang, L. Li, Y. Chen, and D. M. Parkin, "Cancer incidence and mortality estimates and prediction for year 2000 and 2005 in China," Chinese Journal of Health Statistics, vol. 22, no. 4, pp. 218-222, 2005.

[3] Y. He, X. Zheng, C. Sit et al., "Using association rules mining to explore pattern of Chinese medicinal formulae (prescription) in treating and preventing breast cancer recurrence and metastasis," Journal of Translational Medicine, vol. 10, supplement 1, p. S12, 2012.

[4] Z. Chen, L. Y. Chen, P. Wang et al., "Tumor microenvironment varies under different TCM ZHENG models and correlates with treatment response to herbal medicine," Evidence-Based Complementary and Alternative Medicine, vol. 2012, Article ID 635702, 10 pages, 2012.

[5] Q. F. Tang, X. Liu, Y. Ge et al., "Experimental study on inhibiting proliferation and inducing apoptosis of zuo jin wan alcohol extracts on human gastric cancer cells infected by Helicobacter pylori," Chongqing Medicine, vol. 41, no. 15, pp. 1462-1464, 2012.

[6] D. C. Chao, L. J. Lin, S. T. Kao et al., "Inhibitory effects of ZuoJin-Wan and its alkaloidal ingredients on activator protein 1, nuclear factor- $\kappa \mathrm{B}$, and cellular transformation in HepG2 cells," Fitoterapia, vol. 82, no. 4, pp. 696-703, 2011.

[7] H. Sui, X. Liu, B. H. Jin et al., "Zuo Jin Wan, a traditional Chinese herbal formula, reverses P-gp-mediated MDR in vitro and in vivo," Evidence-Based Complementary and Alternative Medicine, vol. 2013, Article ID 957078, 13 pages, 2013.

[8] Xu, Y. Qi, L. Lv et al., "In vitro anti-proliferative effects of Zuojinwan on eight kinds of human cancer cell lines," Cytotechnology, 2013.

[9] Z. Q. Fang, Z. Q. Pan, and W. L. Xu, "Methodology and purposes of establishing mouse and rat models for syndrome differentiation and treatment," Zhong Xi Yi Jie He Xue Bao, vol. 7, no. 10, pp. 907-912, 2009.

[10] X. Zheng, Guideline for Clinical New Drug Research in Chinese Herbal Medicine, vol. 5, Chinese Medical Science and Technology Press, Beijing, China, 2002.

[11] Y. N. Song, J. J. Sun, Y. Y. Lu et al., "Therapeutic efficacy of Fuzheng-Huayu tablet based traditional Chinese medicine syndrome differentiation on hepatitis-B-caused cirrhosis: a multicenter double-blind randomized controlled trail," EvidenceBased Complementary and Alternative Medicine, vol. 2013, Article ID 709305, 8 pages, 2013.

[12] S. B. Su, "Recent advance in ZHENG differentiation research in traditional Chinese medicine," International Journal of Integrative Medicine, vol. 1, no. 7, p. 2013, 2013.

[13] C. Chai, J. Kou, D. Zhu et al., "Mice exposed to chronic intermittent hypoxia simulate clinical features of deficiency of both Qi and Yin syndrome in traditional Chinese medicine," Evidence-Based Complementary and Alternative Medicine, vol. 2011, Article ID 356252, 7 pages, 2011.
[14] H. Y. Dai, P. Wang, L. Feng et al., “The molecular mechanisms of traditional Chinese medicine ZHENG syndromes on pancreatic tumor growth," Integrative Cancer Therapies, vol. 9, no. 3, pp. 291-297, 2010.

[15] R. P. Miller, R. K. Tadagavadi, G. Ramesh, and W. B. Reeves, "Mechanisms of cisplatin nephrotoxicity," Toxins, vol. 2, no. 11, pp. 2490-2518, 2010.

[16] T. Boulikas, "Clinical overview on LipoplatinŮ: a successful liposomal formulation of cisplatin," Expert Opinion on Investigational Drugs, vol. 18, no. 8, pp. 1197-1218, 2009.

[17] S. R. McWhinney, R. M. Goldberg, and H. L. McLeod, "Platinum neurotoxicity pharmacogenetics," Molecular Cancer Therapeutics, vol. 8, no. 1, pp. 10-16, 2009.

[18] I. Cohen, M. Tagliaferri, and D. Tripathy, "Traditional Chinese medicine in the treatment of breast cancer," Seminars in Oncology, vol. 29, no. 6, pp. 563-574, 2002.

[19] A. K. Mukherjee, S. Basu, N. Sarkar, and A. C. Ghosh, "Advances in cancer therapy with plant based natural products," Current Medicinal Chemistry, vol. 8, no. 12, pp. 1467-1486, 2001.

[20] W. L. Hsiao and L. Liu, "The role of traditional Chinese herbal medicines in cancer therapy from TCM theory to mechanistic insights," Planta Medica, vol. 76, no. 11, pp. 1118-1131, 2010.

[21] X. F. Wang, J. Du, T. L. Zhang et al., "Inhibitory effects of PC-SPESII herbal extract on human breast cancer metastasis," Evidence-Based Complementary and Alternative Medicine, vol. 2013, Article ID 894386, 11 pages, 2013.

[22] F.-R. Zhao, H.-P. Mao, H. Zhang et al., "Antagonistic effects of two herbs in Zuojin Wan, a traditional Chinese medicine formula, on catecholamine secretion in bovine adrenal medullary cells," Phytomedicine, vol. 17, no. 8-9, pp. 659-668, 2010.

[23] X. Wang, L. Xu, J. Peng, K. Liu, L. Zhang, and Y. Zhang, "In vivo inhibition of s180 tumors by the synergistic effect of the chinese medicinal herbs coptis chinensis and evodia rutaecarpa," Planta Medica, vol. 75, no. 11, pp. 1215-1220, 2009.

[24] X. N. Wang, X. Han, L.-N. Xu et al., "Enhancement of apoptosis of human hepatocellular carcinoma SMMC-7721 cells through synergy of berberine and evodiamine," Phytomedicine, vol. 15, no. 12, pp. 1062-1068, 2008.

[25] J. Yang, L. Wu, S. Tashino, S. Onodera, and T. Ikejima, “Critical roles of reactive oxygen species in mitochondrial permeability transition in mediating evodiamine-induced human melanoma A375-S2 cell apoptosis," Free Radical Research, vol. 41, no. 10, pp. 1099-1108, 2007.

[26] Z. G. Yang, A. Q. Chen, and B. Liu, "Antiproliferation and apoptosis induced by evodiamine in human colorectal carcinoma cells (COLO-205)," Chemistry and Biodiversity, vol. 6, no. 6, pp. 924-933, 2009.

[27] Q. Wang, Y. Cui, T. Dong, X. Zhang, and K. Lin, "Ethanol extract from a Chinese herbal formula, "zuojin Pill”, inhibit the expression of inflammatory mediators in lipopolysaccharide- stimulated RAW 264.7 mouse macrophages," Journal of Ethnopharmacology, vol. 141, no. 1, pp. 377-385, 2012.

[28] Q. S. Wang, S. L. Ding, and H. P. Mao, "Antidepressant-like effect of ethanol extract from Zuojin Pill, containing two herbal drugs of Rhizoma Coptidis and Fructus Evodiae, is explained by modulating the monoaminergic neurotransmitter system in mice," Journal of Ethnopharmacology, vol. 148, no. 2, pp. 603609, 2013. 


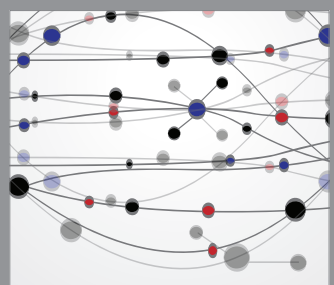

The Scientific World Journal
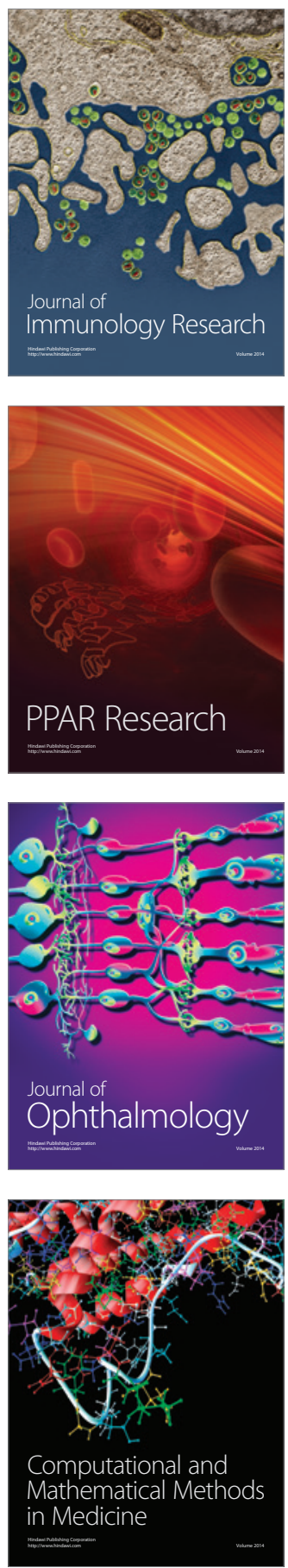

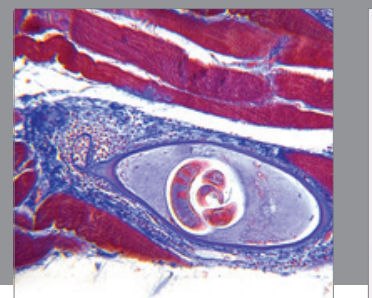

Gastroenterology

Research and Practice
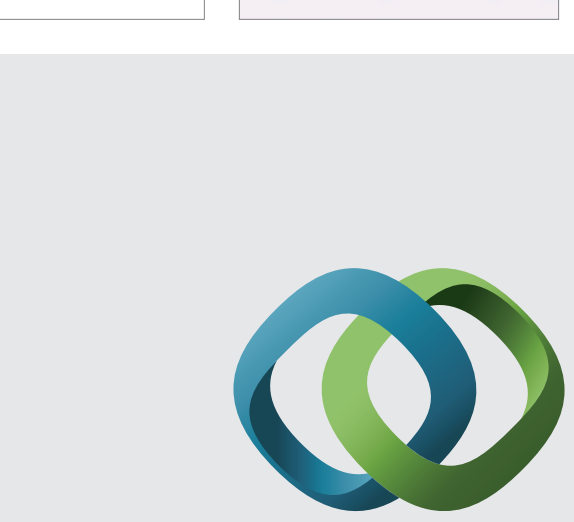

\section{Hindawi}

Submit your manuscripts at

http://www.hindawi.com
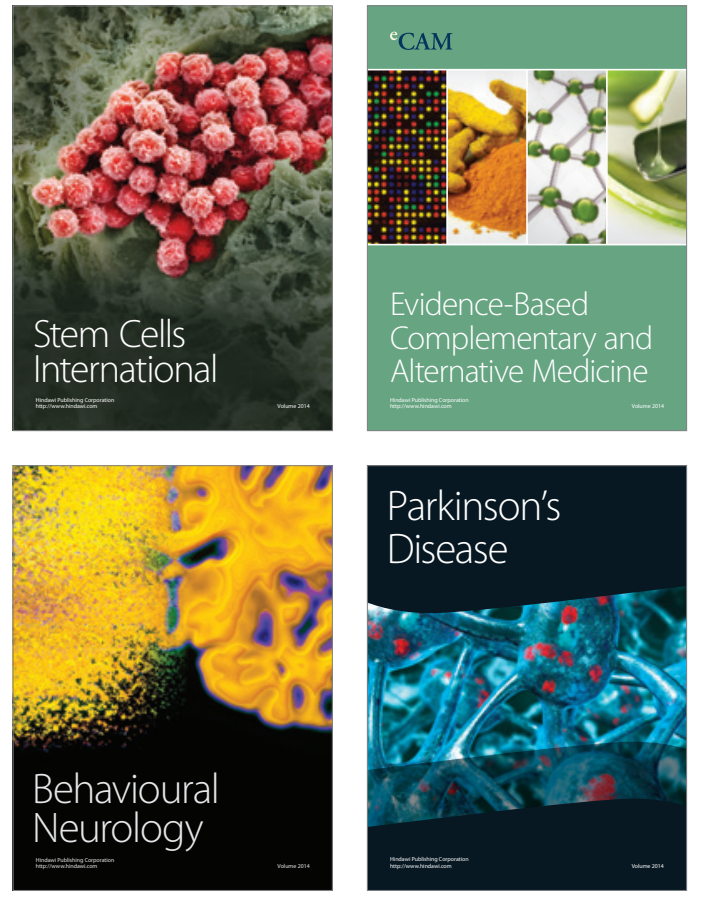
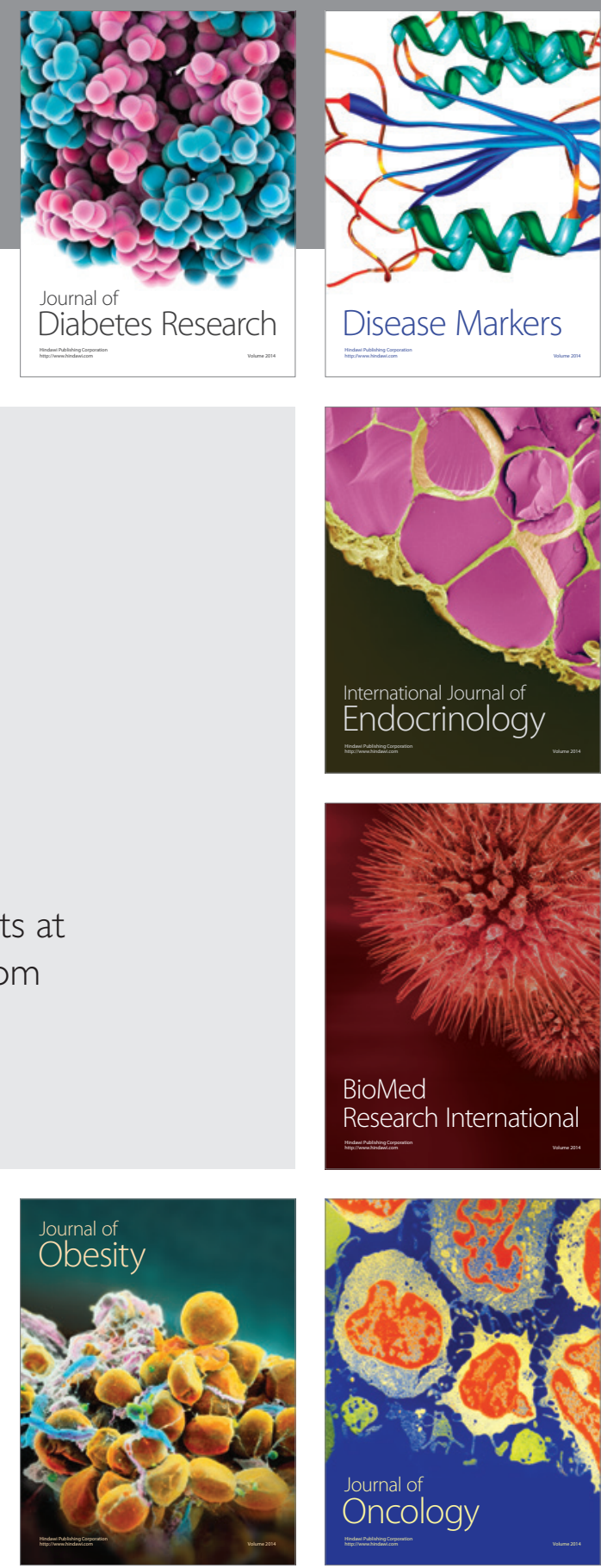

Disease Markers
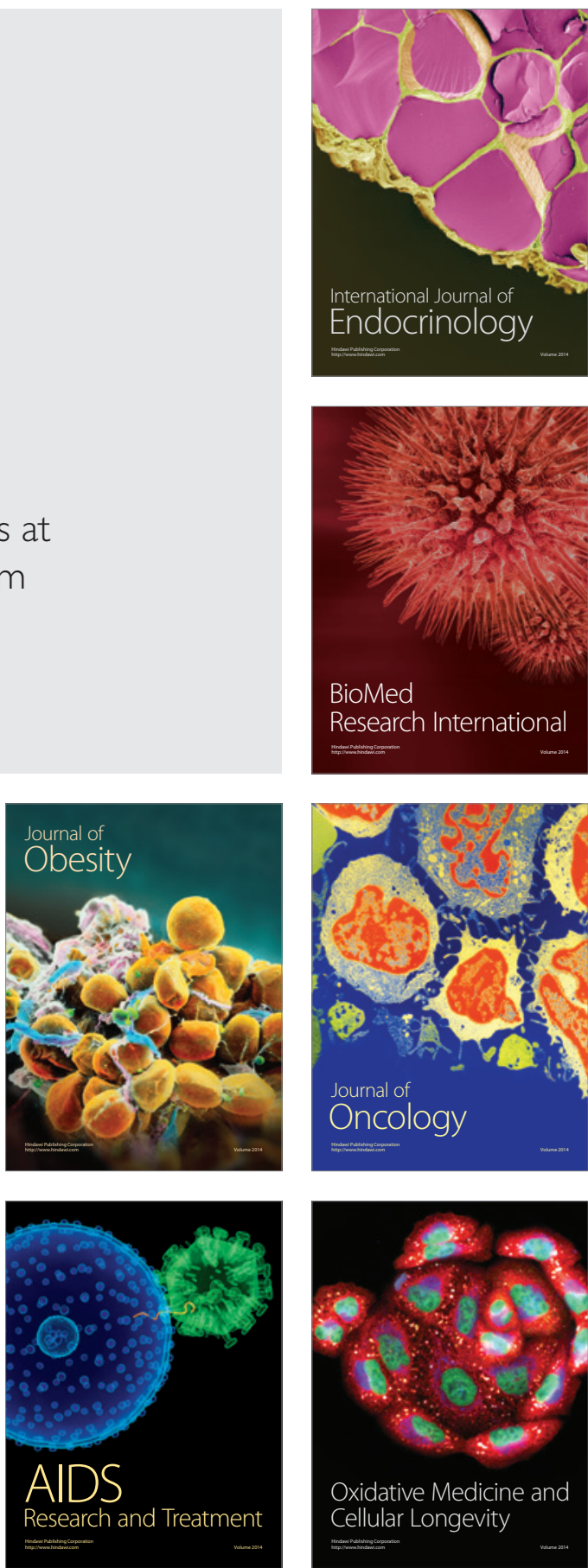